\title{
Hemorrhagic ulcerative uremic stomatitis: a case report
}

wahbi ben salha ${ }^{1}$, eya moussaoui ${ }^{1}$, karim jlassi $^{1}$, lamia oualha ${ }^{1}$, and nabiha douki ${ }^{1}$

${ }^{1}$ University of Monastir Faculty of Dental Medicine of Monastir

November 3, 2021

\begin{abstract}
Uremic stomatitis is a rare manifestation associated with longstanding uremia in chronic renal failure patients. Its evolution is favorable with earlier institution of renal replacement therapy. This case shows that this condition should always be suspected by the dentist when the context of advanced renal disease is present.
\end{abstract}

Title: Hemorrhagic Ulcerative uremic stomatitis: a case report

Ben Salah Wahbi [1,2], Moussaoui eya [1,2], Jlassi karim [1,2], Oualha Lamia [1], Douki Nabiha [1,2]

1: Department of dental medecine, SAHLOUL Hospital (Sousse), Dental Faculty of Monastir, University of Monastir, Tunisia.

2: Laboratory of oral health and maxillofacial rehabilitation (LR12ES11), University of Monastir, Tunisia.

Corresponding author:

Eya MOUSSAOUI : mail address: eyamouss@gmail.com Address: SAHLOUL Hospital, Department of dental medicine, Sousse, Tunisia.

Assistant professor at the department of dental medicine, Department of dental medecine, SAHLOUL Hospital (Sousse), University of Monastir.

Co- authors:

Ben Salha Wahbi: mail address: bensalhawahbi@gmail.com

Resident at the department of dental medicine, Department of dental medecine, SAHLOUL Hospital (Sousse), University of Monastir.

Jlassi karim: mail address: jelassikr7@gmail.com

Resident at the department of dental medicine, Department of dental medecine, SAHLOUL Hospital (Sousse), University of Monastir.

*Lamia OUALHA : mail address: lamia.oualha@gmail.com Address: SAHLOUL Hospital, Department of dental medecine, Sousse, Tunisia.

Professor at the department of dental medicine, SAHLOUL Hospital (Sousse), University of Monastir.

*Nabiha DOUKI : mail address: nabiha.douki@gmail.com Address: SAHLOUL Hospital, Department of dental medecine, Sousse, Tunisia.

Professor and head of department of dental medicine, SAHLOUL Hospital (Sousse), University of Monastir.

Patient consent: 
I confirm that written patient consent has been signed and collected in accordance with the journal's patient consent policy.

\title{
Author contribution:
}

Ben salha wahbi: data collection and interpretation

Eya moussaoui : aquisation and analysis of data and drafting the manuscript

Jlassi karim; collection and interpretation of data

Ouaha lamia: revising the manuscript for important intellectual content

Nabiha douki: conception and design and revising the manuscript

\begin{abstract}
:
Uremic stomatitis is a rare manifestation associated with longstanding uremia in chronic renal failure patients. Its evolution is favorable with earlier institution of renal replacement therapy. This case shows that this condition should always be suspected by the dentist when the context of advanced renal disease is present.
\end{abstract}

Key clinical message: The ulcerative stomatitis should be suspected by the dentist every time the context of end stage renal disease is present even if the patient is under hemodialysis. The high blood uremia, in absence of other possible etiologies will confirm the diagnosis. By decreasing blood urea level, lesions will disappear.

Keywords: uremic stomatitis, uremia, oral ulcerations, chronic renal failure,

ulcerative stomatitis, hemodialysis, hemorrhagic stomatitis

Manuscript:

A 50-year-old woman was referred to the department of dentistry complaining of xerostomia and mouth burning on her tongue. The patient suffered from anorexia and dysphagia. The patient's medical history included chronic renal failure in the hemodialysis stage. She started hemodialysis 2 months ago with one session per week.

Extraoral examination showed ulcerative, hemorrhagic and crusting lesions localized on the nostril rim, philtrum and superior lip (Figure 1). Intraoral examination revealed the presence of multiple hemorrhagic ulcerations involving the dorsal surface of the tongue, buccal mucosa and mucous surfaces of the lips. Lesions were covered by an adherent thick white-yellowish membrane (Figure 2).

Laboratory studies showed a creatine level of $711 \mu \mathrm{mol} / \mathrm{L}$ and a blood urea nitrogen level of more than 32 $\mathrm{mmol} / \mathrm{L}$. A diagnosis of hemorrhagic ulcerative uremic stomatitis was made. Hemodialysis sessions were doubled and partially resolution of oral mucosal lesions was seen after 10 days.

Uremic stomatitis is a rare manifestation associated with longstanding uremia in chronic renal failure patients [1]. Its evolution is favorable with earlier institution of renal replacement therapy [2]. This case shows that this condition should always be suspected by the dentist when the context of advanced renal disease is present.

[1] Barie E. De la stomatite uremique. Arch Gen Med 1889;2:415-32.

[2] Liao C-Y, Wu C-C, Chu P-L. Uremic stomatitis. QJM 2017;110:247-8.

\section{Conflicts of interest:}

All the authors declare not having any conflicts of interest.

\section{Ethics statement:}


Data from the patient included in this case report were treated anonymously and a statement of informed consent was signed to allow the use of her medical and dental records and photos.

\section{Funding:}

The authors received no financial support for the research, authorship, and/or publication of this article.

\section{Hosted file}

DSC_0735.pdf available at https://authorea.com/users/441102/articles/544099-hemorrhagiculcerative-uremic-stomatitis-a-case-report

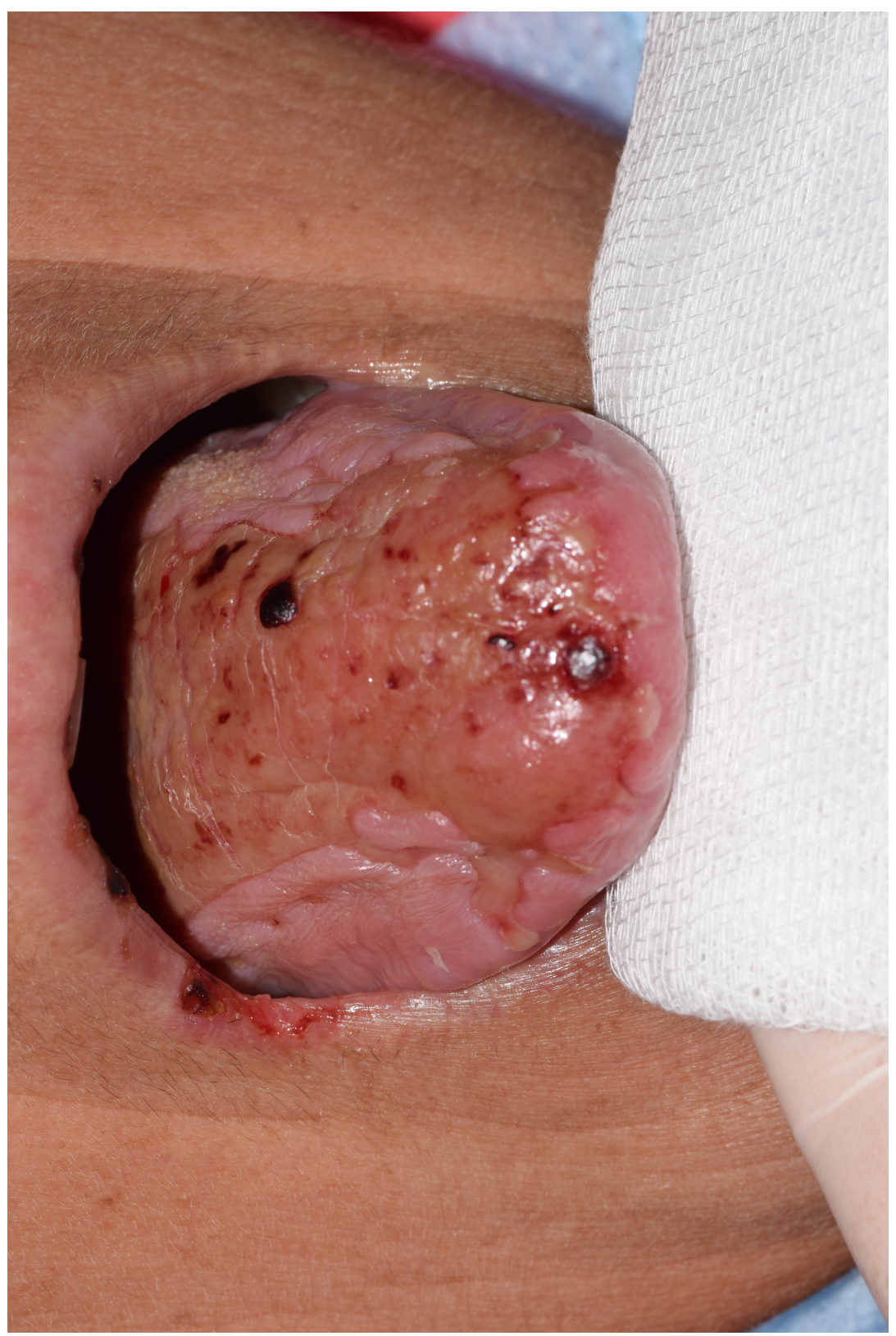

